\title{
Resveratrol prevents osteoporosis in ovariectomized rats by regulating microRNA-338-3p
}

\author{
DA-WEI GUO ${ }^{1,2}$, YA-XIN HAN ${ }^{1}$, LIN CONG $^{1}$, DONG LIANG ${ }^{1}$ and GUAN-JUN TU ${ }^{1}$ \\ ${ }^{1}$ Department of Orthopaedics, The First Affiliated Hospital of China Medical University, Shenyang, Liaoning 110001; \\ ${ }^{2}$ Department of Orthopaedics, The 4th People's Hospital of Shenyang, Shenyang, Liaoning 110000, P.R. China
}

Received June 18, 2014; Accepted March 4, 2015

DOI: $10.3892 / \mathrm{mmr} .2015 .3581$

\begin{abstract}
Osteoporosis is a disease characterized by loss of bone mass and degeneration of the microstructure of bone. Resveratrol (3,5,4-tri-hydroxystilbene; RESV) may delay the onset of a variety of age-related diseases. In the present study, an ovariectomized female rat model was used to detect the changes in microRNAs (miRNAs/miRs) following RESV treatment. Subsequently, the target genes of miRNA were predicted using TargetScan software and determined using a dual-luciferase reporter assay. Finally, the role of miR-338-3p in the proliferation and differentiation of human osteoblast (HOB) cells was confirmed. The predominant finding of the present study was the identification of an intact mechanism of the effect of RESV in osteoporosis treatment. The results suggested that RESV suppresses miR-338-3p, followed by an increase in the expression of runt-related transcription factor 2 in HOB cells.
\end{abstract}

\section{Introduction}

Osteoporosis is a disease characterized by loss of bone mass and degeneration of the bone microstructure (1). It is estimated that it affects one in two women $>50$ years of age in the UK (2). Osteoporosis also represents a major public health concern in Asian countries, particularly China (3), resulting in a requirement for investigation into osteoporosis treatment methodology.

Resveratrol (3,5,4-tri-hydroxystilbene; RESV) is synthesized by various species of plant (4). It is a phytoalexin antimicrobial, which has broad-spectrum beneficial health effects, including anti-infective, antioxidant and cardioprotective functions (5). Furthermore, RESV has demonstrated

Correspondence to: Professor Guan-Jun Tu, Department of Orthopaedics, The First Affiliated Hospital of China Medical University, 155 Nanjing North, Shenyang, Liaoning 110001, P.R. China

E-mail: tu_guanj@163.com

Key words: ovariectomized rats, resveratrol, microRNA-338-3p, runt-related transcription factor 2, osteoporosis promise in delaying the onset of a variety of age-related diseases (6). RESV has been revealed to stimulate bone cell proliferation and differentiation (7). Liu et al (8) demonstrated that RESV increases the bone mineral density (BMD) of the epiphyses of ovariectomized (OVX) female rats. However, further investigation is required in order to fully elucidate the mechanisms underlying the effects of RESV in the treatment of osteoporosis. MicroRNAs (miRNA/miR) are short, single-stranded RNAs consisting of 20-25 nucleotides. They are able to bind complementary sequences in the 3'-untranslated regions (UTR) of target genes to induce mRNA degradation or suppress translation (9).

The purpose of the present study was to determine the effects of RESV on BMD and the microarchitecture of the bone in OVX rats, and examine their effects on the bone by investigating the expression of bone-specific genes. In addition, the mechanisms underlying the effects of RESV were determined.

\section{Materials and methods}

Animals. The experiments were performed using 200 8-week-old female Wistar rats (BetterBiotechnology Co., Ltd., Nanjing, China) weighing between 180 and $200 \mathrm{~g}$. Throughout the experiment, the animals were housed in groups in a room with controlled temperature $\left(22^{\circ} \mathrm{C}\right)$ and humidity $(50 \%)$ with a $12 \mathrm{~h}$ light-12 h dark cycle. Standard rat chow (BetterBiotechnology Co., Ltd.) and tap water were available ad libitum. All procedures involving the animals were approved by the ethics committee of China Medical University (Shenyang, China).

OVX and treatment. The OVX was performed under isoflurane $(10 \%, 0.35 \mathrm{ml} / 100 \mathrm{~g})$ anesthesia. A single dorsal incision was made in the skin and, through this, two lateral incisions were made in the muscle layer $\sim 0.5 \mathrm{~cm}$ below each kidney. The ovaries were extruded through the incision, ligated off and removed. At 20 days post-surgery, a $500 \mathrm{mg} / \mathrm{kg}$ dose of RESV was prepared by mixing RESV (Sigma-Aldrich, St. Louis, MO, USA) with deionized water at a ratio of 3:7, and was administered to the OVX female rats through tail intravenous injection. The OVX rats were divided into four groups $(n=10$ per group) as follows: Untreated rats; OVX rats OVX rats with phosphate-buffered saline (PBS; Sigma-Aldrich); and OVX 
rats with RESV. The subcutaneous fat and the uterus were also harvested, rinsed with physiological saline, and weighed.

$B M D$. The BMD was assessed using dual-energy $\mathrm{X}$-ray absorptiometry with a Hologic QDR-4500A X-ray bone densitometer (Hologic, Inc., Danbury, CT, USA). The total body BMD was assessed on the 30th and 60th day following the administration of RESV.

Bone turnover markers in serum and urine. The concentrations of calcium, phosphorus and creatinine in the serum and urine were measured using a commercial kit (Quanti Chrom ${ }^{\mathrm{TM}}$ Bioassay Systems, Hayward, CA, USA). The levels of serum alkaline phosphatase (ALP) and osteocalcin were measured using a commercial ALP kit (Asan Pharm, Seoul, Korea) and Rat-MID ${ }^{\text {тм }}$ Osteocalcin EIA kit (Immunodiagnostic systems Inc., Stoughton, MA, USA), respectively. The level of urinary deoxypyridinoline (DPD) was measured using a METRA ${ }^{\mathrm{TM}}$ DPD EIA kit (Quidel Corporation, San Diego, CA, USA). All experiments were performed according to the manufacturer's instructions and the results were analyzed using a microplate reader (Bio-Rad Laboratories, Inc., Richmond, CA, USA). The values for DPD were adjusted for volume by determining the level of urinary creatinine (Quanti Chrom ${ }^{\mathrm{TM}}$ Bioassay Systems), with concentrations presented as nmol DPD/mmol creatinine.

miRNA expression array. Total RNA was extracted using TRIzol reagent (Invitrogen Life Technologies, Carlsbad, CA, USA), and further purified using an mirVana miRNA isolation kit (Life Technologies, Grand Island, NY, USA). Microarray analysis was performed using an Agilent Whole Rat Genome Array (Agilent, Santa Clara, CA, USA), according to the manufacturer's instructions. The integrity of the total RNA was assessed using an Agilent 2100 Bioanalyzer (Agilent). cDNA was synthesized from RNA by reverse transcription using the PrimeScript ${ }^{\mathrm{TM}}$ II 1st strand cDNA synthesis kit (cat. no. 6210B; Takara Bio Inc., Dalian, China). The cDNA and biotinylated cRNA were synthesized and hybridized to the array. The slides were scanned using an Agilent Microarray Scanner (cat no. G2565BA; Agilent) and Feature Extraction software 10.7 (Agilent) with default settings. The raw data were normalized by Quantile algorithm, Gene Spring software 11.0 (Agilent).

Western blot analysis. The rats were sacrificed using ketamine $(70 \mathrm{mg} / \mathrm{kg})$ and xylazine $(6 \mathrm{mg} / \mathrm{kg})$ purchased from Sigma-Aldrich. The femur tissues were obtained from the rat models and lysed in $20 \mathrm{mM}$ Tris- $\mathrm{HCl}$ buffer ( $\mathrm{pH} 7.4)$, containing $150 \mathrm{mM} \mathrm{NaCl}, 2 \mathrm{mM}$ EDTA, $1 \%$ Nonidet P-40, $50 \mathrm{mM} \mathrm{NaF}, 1 \mathrm{mM} \mathrm{Na} \mathrm{VO}_{4}, 1 \mathrm{mM} \mathrm{Na}{ }_{2} \mathrm{MoO}_{4}, 10 \mu \mathrm{m}$ leupeptin (Roche, Basel, Switzerland). The protein concentration was quantified using a bicinchoninic protein assay kit (CW Biotech, Beijing, China). The crude lysates were then centrifuged at $14,000 \mathrm{x}$ g for $10 \mathrm{~min}$, following which the cleared lysates were collected and were separated by 8\% SDS-polyacrylamide gel electrophoresis (Sigma-Aldrich) and transferred onto nitrocellulose membranes (Bio-Rad Laboratories, Inc., Hercules, CA, USA). The membranes were blocked with $5 \%$ milk-Tris-buffered saline with
5\% Tween-20 (Sigma-Aldrich) and incubated with primary antibodies at room temperature overnight. Rabbit polyclonal anti-runt-related transcription factor (RUNX)2 (1:200; cat. no. sc-10758) and mouse monoclonal anti- $\beta$-actin (1:200; cat. no. sc-47778) antibodies were purchased from Santa Cruz Biotechnology, Inc. (Santa Cruz, CA, USA). The secondary antibodies were purchased from Beyotime Biotechnology (Beijing, China). The secondary antibodies included anti mouse IgG (cat. no. A0216), anti rabbit IgG (cat. no. A0239) or anti goat IgG (cat. no. A0181). The membranes were incubated with the secondary antibodies for $2 \mathrm{~h}$ at room temperature. The membranes were then developed and visualized using a Pierce ECL kit (Thermo Fisher Scientific Inc., Waltham, MA, USA). Densitometric quantification for western blotting was performed using Scion 4.03 image software (Scion Corporation, Bethesda, MD, USA).

Target gene prediction. The target gene information of mmu-miR-338-3p was analyzed using microRNA TargetScan software (http://www.microrna.org/mammalian/index.html). The minimum free energy predicted for hybridization was determined by BibiServ analysis (http://bibiserv.techfak. uni-bielefeld.de/genefisher2).

Cell lines. Human osteoblast (HOB) cells were purchased from the Shanghai Institutes for Biological Sciences, Chinese Academy of Sciences (Shanghai, China) and maintained in osteoblast basal media (Lonza, Walkersville, MD, USA) supplemented with fetal bovine serum (Lonza).

Transfection. The anti-miRNA inhibitor (anti-miR-338-3p) and negative control miRNA (anti-control) were purchased from Ambion Life Technologies, Carlsbad, CA, USA). For transfection, the cells were plated at 70-80\% confluency in a 6-well dish. After $24 \mathrm{~h}$ at room temperature, the cells were transfected with $1 \mu \mathrm{g}$ chemically-synthesized RNA (GenePharma Co., Ltd., Shanghai, China) using Lipofectamine 2000 (Invitrogen Life Technologies), according to the manufacturer's instructions.

Immunofluorescence. The cells were washed with PBS, fixed with $4 \%$ formaldehyde in PBS for $20 \mathrm{~min}$, and finally permeabilized with $0.2 \%$ Triton $\mathrm{X}-100$ solution (Beyotime Biotechnology) for $5 \mathrm{~min}$ at room temperature. The cells were incubated with anti-RUNX2 antibody for $1 \mathrm{~h}$, washed three times with PBS and then incubated with the appropriate Alexa Fluor 594-conjugated secondary antibody The cells were then washed three times in PBS. The slides were mounted with VECTASHIELD mounting medium (Vector Laboratories, Inc., Burlingame, CA, USA). For every coverslip, the cells were visualized and images were captured in five random fields using an Olympus CX71 fluorescence microscope (Olympus Corporation, Tokyo, Japan).

Cell proliferation. The HOBs were plated at a density of $1 \times 10^{3}$ cells/well in 96-well plates. The mitogenic activity following treatment with RESV was assayed using a colorimetric 3-(4, 5-dimethylthiazol-2-yl)-5-(3-carboxymetho xyphenyl)-2-(4-sulfophenyl)-2H-tetrazolium (MTS) assay (Promega, Madison, WI, USA). Following treatment of the 
Table I. Effects in of OVX and RESV treatment in rats on body weight $(\mathrm{g})$.

\begin{tabular}{lcccr}
\hline Group & $\mathrm{n}$ & Day 0 & Day 30 & Day 60 \\
\hline Untreated & 10 & $203.26 \pm 2.45$ & $222.27 \pm 2.09$ & $238.35 \pm 2.72$ \\
OVX & 10 & $201.85 \pm 1.98$ & $252.46 \pm 1.93$ & $302.24 \pm 3.48$ \\
OVX + PBS & 10 & $198.42 \pm 2.17$ & $261.33 \pm 2.21$ & $292.67 \pm 3.22$ \\
OVX + RESV & 10 & $202.67 \pm 2.24$ & $231.92 \pm 2.87$ & $255.24 \pm 1.88$
\end{tabular}

Data are presented as the mean \pm standard deviation. OVX, ovariectomized; PBS, phosphate-buffered saline; RESV, resveratrol.

Table II. Effects in of OVX and RESV treatment in rats on levels of subcutaneous fat (g).

\begin{tabular}{lccrr}
\hline Group & $\mathrm{n}$ & Day 0 & Day 30 & Day 60 \\
\hline Untreated & 10 & $3.75 \pm 0.23$ & $3.80 \pm 0.32$ & $4.02 \pm 0.31$ \\
OVX & 10 & $3.64 \pm 0.25$ & $4.81 \pm 0.43$ & $6.92 \pm 0.46$ \\
OVX + PBS & 10 & $3.63 \pm 0.34$ & $4.77 \pm 0.31$ & $6.56 \pm 0.28$ \\
OVX + RESV & 10 & $3.76 \pm 0.29$ & $4.09 \pm 0.21$ & $4.27 \pm 0.37$
\end{tabular}

Data are presented as the mean \pm standard deviation. OVX, ovariectomized; PBS, phosphate-buffered saline; RESV, resveratrol.

different groups, $20 \mu 1$ MTS was added to each well for $4 \mathrm{~h}$ at room temperature, and the light absorbance at $490 \mathrm{~nm}$ was measured using a spectrophotometric microplate reader (Model 680; Bio-Rad Laboratories, Inc.).

In vitro mineralization assay. The cells were seeded in 6-well plates, in triplicate, at a density of $3 \times 10^{3} / \mathrm{cm}^{2}$. Alizarin Red S staining, which detects calcium deposition, was used as an indicator of mineralization. The cells were rinsed in PBS and fixed in $70 \%$ ice-cold ethanol prior to staining with $40 \mathrm{mM}$ Alizarin Red S (pH 4.2; Sigma-Aldrich) for $10 \mathrm{~min}$ at room temperature. The calcium content was then quantified by measuring the level of Alizarin Red S staining, which was bound to the mineralizing nodules.

Dual-luciferase reporter assay. Dual-luciferase reporter assays were performed in the HOB cells to determine the binding site of $m i R-338-3 p$ within the 3'UTR of RUNX2. Briefly, a pMIR-reporter (Signosis, Inc., Santa Clara, CA, USA), containing either the wild-type or mutant 3'-UTR was cotransfected with phRL-TK (Signosis, Inc.) into the HOB cells. At $48 \mathrm{~h}$ post-transfection, the cells were harvested and analyzed using a dual-luciferase reporter assay (Promega), according to the manufacturer's instructions. Briefly, the cell extract was subjected to dual-luciferase assay as measured by the Xenogen IVIS 200 imaging system (Caliper Life Sciences, Hopkinton, MA, USA). The data were presented as luciferase activity/RL-TK activity.

Reverse transcription-quantitative polymerase chain reaction $(q P C R)$. qPCR was performed using specific primers. qPCR analysis was performed on an ABI prism 7500 sequence detection system (Applied Biosystems, Foster City, CA, USA), using SYBR Green PCR master mixture (Takara Bio Inc.). The PCR conditions were as follows: One cycle

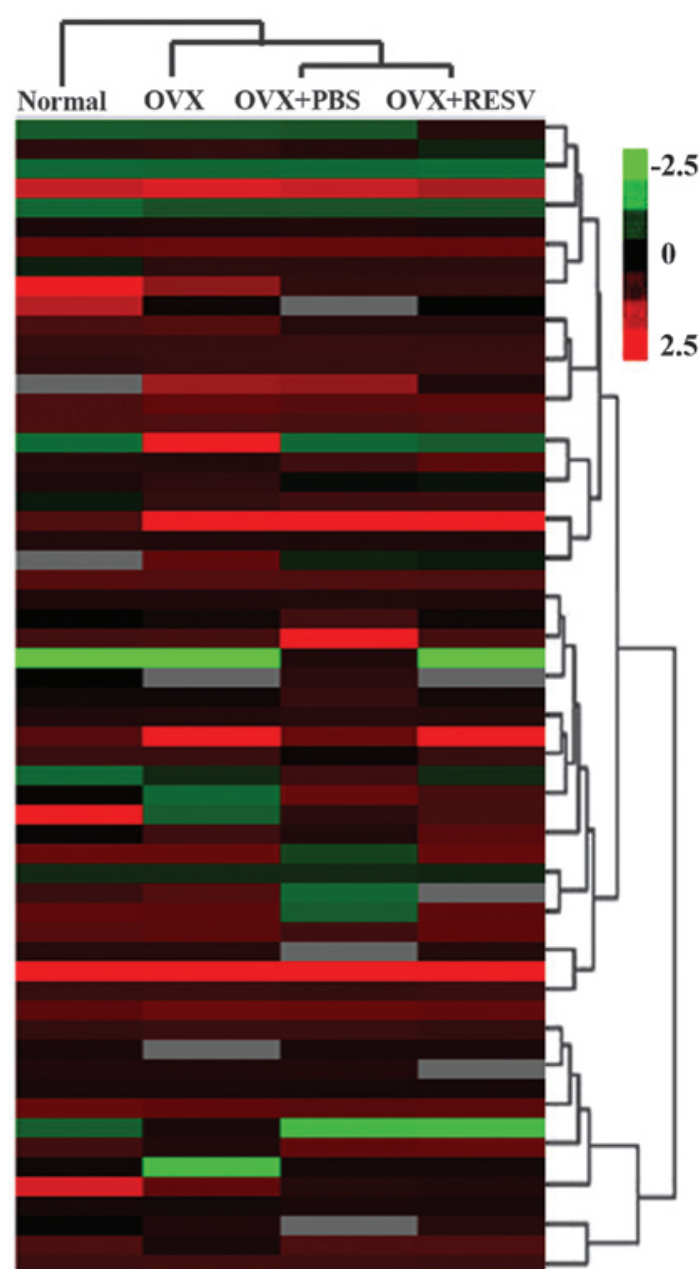

Figure 1. Microarray analysis revealed numerous subtle differences in the expression levels of microRNA in OVX rats following treatment with RESV. Intensities are mean centered, with red indicating higher values and green indicating lower values. RESV, resveratrol; OVX, ovariectomized; PBS, phosphate-buffered saline. 
Table III. Effects of OVX and RESV treatment on uterus weight (mg).

\begin{tabular}{lcccc}
\hline Group & $\mathrm{n}$ & Day 0 & Day 30 & Day 60 \\
\hline Untreated & 10 & $497.56 \pm 25.96$ & $500.42 \pm 22.58$ & $503.57 \pm 22.53$ \\
OVX & 10 & $498.39 \pm 29.03$ & $271.85 \pm 14.14$ & $89.86 \pm 4.78$ \\
OVX + PBS & 10 & $500.91 \pm 27.35$ & $288.45 \pm 12.35$ & $95.67 \pm 5.28$ \\
OVX + RESV & 10 & $494.75 \pm 25.75$ & $342.35 \pm 13.88$ & $309.43 \pm 12.94$
\end{tabular}

Data are presented as the mean \pm standard deviation. OVX, ovariectomized; PBS, phosphate-buffered saline; RESV, resveratrol.

Table IV. Effects of OVX and RESV treatment on bone mineral densities of the total body $\left(\mathrm{mg} / \mathrm{cm}^{2}\right)$.

\begin{tabular}{lcccr}
\hline Group & $\mathrm{n}$ & Day 0 & Day 30 & Day 60 \\
\hline Untreated & 10 & $161.58 \pm 11.24$ & $178.89 \pm 10.79$ & $196.49 \pm 13.52$ \\
OVX & 10 & $159.23 \pm 10.25$ & $147.42 \pm 10.65$ & $131.26 \pm 11.29$ \\
OVX + PBS & 10 & $160.76 \pm 13.36$ & $145.36 \pm 11.44$ & $134.95 \pm 11.10$ \\
OVX + RESV & 10 & $158.64 \pm 10.21$ & $152.27 \pm 10.38$ & $148.62 \pm 12.93$ \\
\hline
\end{tabular}

Data are presented as the mean \pm standard deviation. OVX, ovariectomized; PBS, phosphate-buffered saline; RESV, resveratrol.

Table V. Effects of OVX and RESV treatment on levels of serum calcium (mmol/l).

\begin{tabular}{lcccr}
\hline Group & $n$ & Day 0 & Day 30 & Day 60 \\
\hline Untreated & 10 & $6.24 \pm 0.31$ & $6.31 \pm 0.32$ & $6.32 \pm 0.27$ \\
OVX & 10 & $6.33 \pm 0.32$ & $5.12 \pm 0.28$ & $4.74 \pm 0.26$ \\
OVX + PBS & 10 & $6.18 \pm 0.27$ & $5.04 \pm 0.25$ & $4.66 \pm 0.21$ \\
OVX + RESV & 10 & $6.27 \pm 0.28$ & $5.69 \pm 0.34$ & $5.08 \pm 0.35$
\end{tabular}

Data are presented as the mean \pm standard deviation. OVX, ovariectomized; PBS, phosphate-buffered saline; RESV, resveratrol.

Table VI. Effects of OVX and RESV treatment on levels of serum phosphorus (mmol/l).

\begin{tabular}{lcccr}
\hline Group & $n$ & 0 day & Day 30 & Day 60 \\
\hline Untreated & 10 & $4.72 \pm 0.57$ & $4.84 \pm 0.53$ & $4.72 \pm 0.44$ \\
OVX & 10 & $4.83 \pm 0.48$ & $5.22 \pm 0.41$ & $5.71 \pm 0.46$ \\
OVX + PBS & 10 & $4.68 \pm 0.53$ & $5.32 \pm 0.45$ & $5.69 \pm 0.47$ \\
OVX + RESV & 10 & $4.71 \pm 0.47$ & $4.97 \pm 0.86$ & $5.02 \pm 0.46$
\end{tabular}

Data are presented as the mean \pm standard deviation. OVX, ovariectomized; PBS, phosphate-buffered saline; RESV, resveratrol.

at $95^{\circ} \mathrm{C}$ for $10 \mathrm{~min}$ followed by 40 cycles at $95^{\circ} \mathrm{C}$ for $15 \mathrm{sec}$ and at $60^{\circ} \mathrm{C}$ for $1 \mathrm{~min}$. The following primer sets were used: $R U N X 2$, sense 5'-ATGGCCTGGTCCATCTCCAC-3' and antisense 5'-GGCAGAGGTGAAAAAGTTGC-3' and GAPDH, sense 5'-CACTGGCGGTGCAACAAGA-3' and antisense 5'-CATGGGTGGAATCATATTGGAA-3'. The $m i R N A-338-3 p$ and $U 6$ primers were purchased from Qiagen (Hilden, Germany). Relative quantification was calculated using the $2^{-\Delta \Delta \mathrm{Ct}}$ method, where $\Delta \Delta \mathrm{Ct}=\mathrm{Ct}(\mathrm{RUNX} 2)-\mathrm{Ct}(\mathrm{GA}$
PDH) (10). The expression level of the control group was considered "1".

Multiple sequence alignment and phylogenetic analysis. A total of 19 nucleotide sequences of $m i R-338-3 p$ from a wide range of organisms were obtained from the National Center for Biotechnology Information database (https://www. ncbi.nlm.nih.gov/). Multiple sequence alignments were performed using the ClustalW programme (11). To determine 
Table VII. Effects of OVX and RESV treatment on levels of serum alkaline phosphatase (U/1).

\begin{tabular}{lcccr}
\hline Group & $\mathrm{n}$ & Day 0 & Day 30 & Day 60 \\
\hline Untreated & 10 & $11.49 \pm 1.64$ & $11.54 \pm 1.79$ & $11.52 \pm 1.57$ \\
OVX & 10 & $11.65 \pm 1.57$ & $17.53 \pm 1.84$ & $21.28 \pm 1.99$ \\
OVX + PBS & 10 & $10.34 \pm 1.65$ & $18.85 \pm 1.41$ & $22.05 \pm 1.57$ \\
OVX + RESV & 10 & $11.75 \pm 1.61$ & $14.27 \pm 1.62$ & $16.26 \pm 1.93$ \\
\hline
\end{tabular}

Data are presented as the mean \pm standard deviation. OVX, ovariectomized; PBS, phosphate-buffered saline; RESV, resveratrol.

Table VIII. Effects of OVX and RESV treatment on levels of serum osteocalcin (nmol/l).

\begin{tabular}{lcccr}
\hline Group & $\mathrm{n}$ & Day 0 & Day 30 & Day 60 \\
\hline Untreated & 10 & $2.72 \pm 0.37$ & $2.86 \pm 0.38$ & $2.85 \pm 0.41$ \\
OVX & 10 & $2.82 \pm 0.38$ & $2.27 \pm 0.35$ & $1.71 \pm 0.42$ \\
OVX + PBS & 10 & $2.58 \pm 0.41$ & $2.32 \pm 0.41$ & $1.89 \pm 0.38$ \\
OVX + RESV & 10 & $2.57 \pm 0.35$ & $2.48 \pm 0.48$ & $2.52 \pm 0.47$ \\
\hline
\end{tabular}

Data are presented as the mean \pm standard deviation. OVX, ovariectomized; PBS, phosphate-buffered saline; RESV, resveratrol.

Table IX. Effects of OVX and RESV treatment on levels of urinary calcium ( $\mathrm{mmol} / \mathrm{l})$.

\begin{tabular}{lcccr}
\hline Group & $\mathrm{n}$ & Day 0 & Day 30 & Day 60 \\
\hline Untreated & 10 & $1.68 \pm 0.29$ & $1.76 \pm 0.28$ & $1.65 \pm 0.31$ \\
OVX & 10 & $1.72 \pm 0.34$ & $2.65 \pm 0.43$ & $2.71 \pm 0.54$ \\
OVX + PBS & 10 & $1.58 \pm 0.22$ & $2.68 \pm 0.47$ & $2.84 \pm 0.56$ \\
OVX + RESV & 10 & $1.66 \pm 0.27$ & $2.01 \pm 0.48$ & $2.32 \pm 0.35$ \\
\hline
\end{tabular}

Data are presented as the mean \pm standard deviation. OVX, ovariectomized; PBS, phosphate-buffered saline; RESV, resveratrol.

Table X. Effects of OVX and RESV treatment on levels of urinary deoxypyridinoline/creatinine (nmol/mmol).

\begin{tabular}{lcccr}
\hline Group & $\mathrm{n}$ & Day 0 & Day 30 & Day 60 \\
\hline Untreated & 10 & $147.25 \pm 10.09$ & $148.75 \pm 10.82$ & $150.50 \pm 11.31$ \\
OVX & 10 & $129.75 \pm 10.85$ & $222.24 \pm 14.42$ & $271.52 \pm 15.88$ \\
OVX + PBS & 10 & $130.46 \pm 11.14$ & $225.36 \pm 14.47$ & $274.98 \pm 14.92$ \\
OVX + RESV & 10 & $148.89 \pm 11.33$ & $191.67 \pm 14.24$ & $207.68 \pm 14.45$ \\
\hline
\end{tabular}

Data are presented as the mean \pm standard deviation. OVX, ovariectomized; PBS, phosphate-buffered saline; RESV, resveratrol.

the phylogenetic associations between these sequences, maximum likelihood, neighbor-joining and Bayesian Markov chain Monte Carlo approaches were used to infer three individual trees.

Statistical analysis. The data are presented as the mean \pm standard deviation Statistical analysis was performed using a two-tailed t-test using SPSS 17.0 statistical software (SPSS, Inc., Chicago, IL, USA). P $<0.05$ was considered to indicate a statistically significant difference. All experiments were repeated at least three times.

\section{Results}

Body weight and uterus weight. The baseline body weights of the animal groups were similar (Table I), however, the final body weights and subcutaneous fat weights were significantly higher in the OVX group and the OVX + PBS groups, compared with the 
A

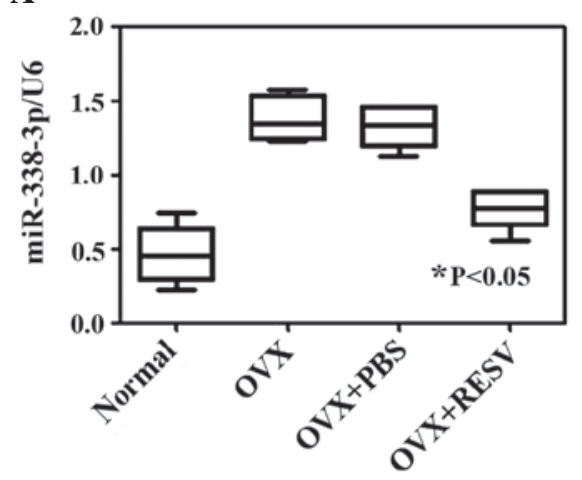

B

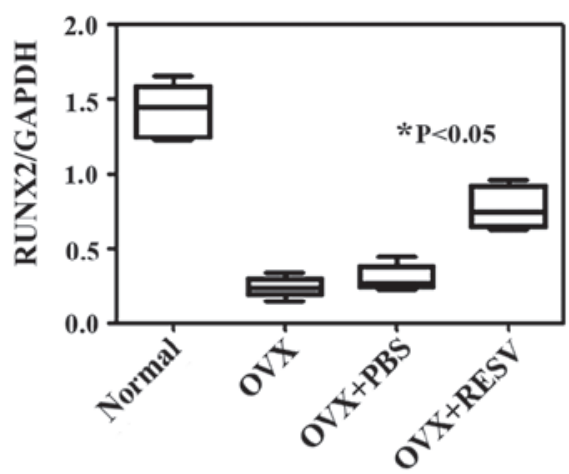

C

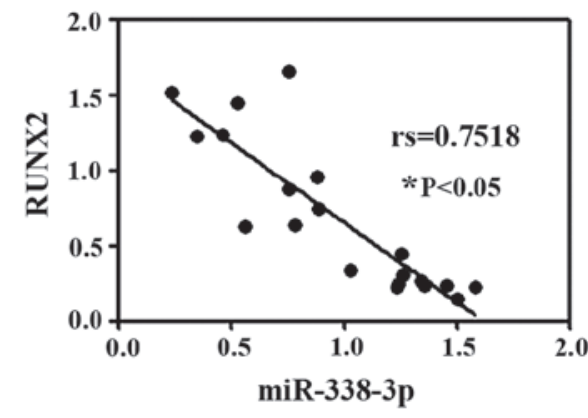

D
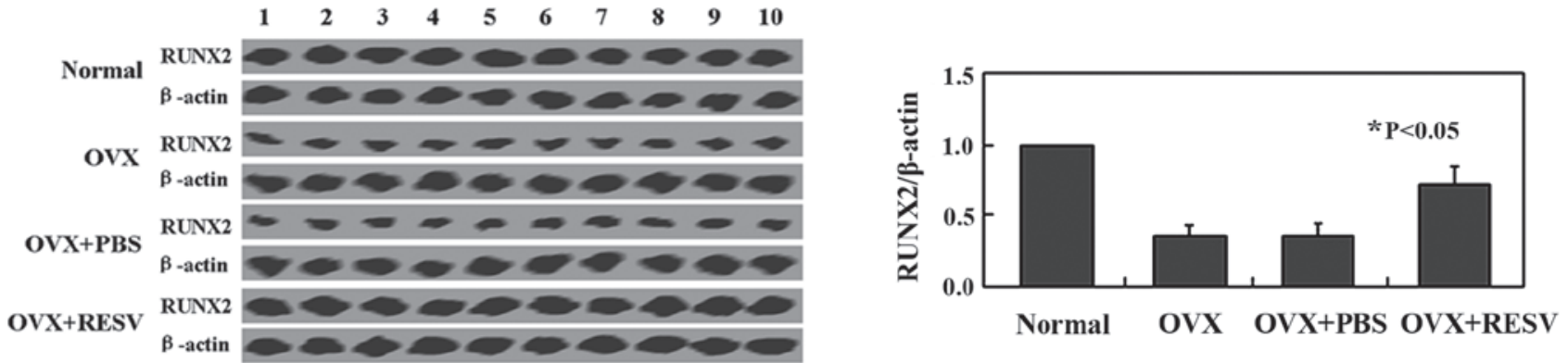

Figure 2. Expression levels of miR-338-3p and RUNX2 were measured in OVX rats. (A) Levels of miR-338-3p were determined by qPCR. U6 was used as an internal control. (B) Levels of RUNX2 were determined by qPCR. GAPDH was used as an internal control. (C) Negative correlation was observed between the mRNA expression levels of $m i R-338-3 p$ and RUNX2. (D) Protein expression levels of RUNX2 were determined using western blot analysis. Ten different rats were randomly selected from each group. The expression of $\beta$-actin was used as an internal control. Data are presented as the mean \pm standard deviation. ${ }^{*} \mathrm{P}<0.05$, vs. the control group. miR, microRNA; RUNX2, runt-related transcription factor 2; qPCR, quantitative polymerase chain reaction; RESV, resveratrol; OVX, ovariectomized; PBS, phosphate-buffered saline.

A

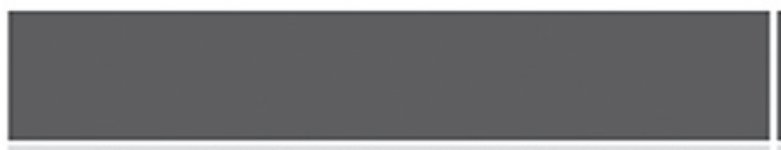

Position 1232-1239 of RUNX2 3' UTR m mu-m iR-338-3p predicted consequential pairing of target region (top) and miRNA (bottom) 5' ... CUUUACUAGCAUGAAAUGCUGGA. . . $3^{\prime}$

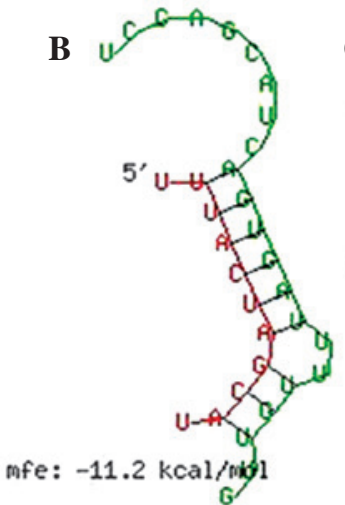

C

RUNX2-Mut+ mmu-miR-338-3p

RUNX2 -Mut+NC

RUNX2 -WT + mmu-miR-338-3p

RUNX2 - WT +NC

0.0

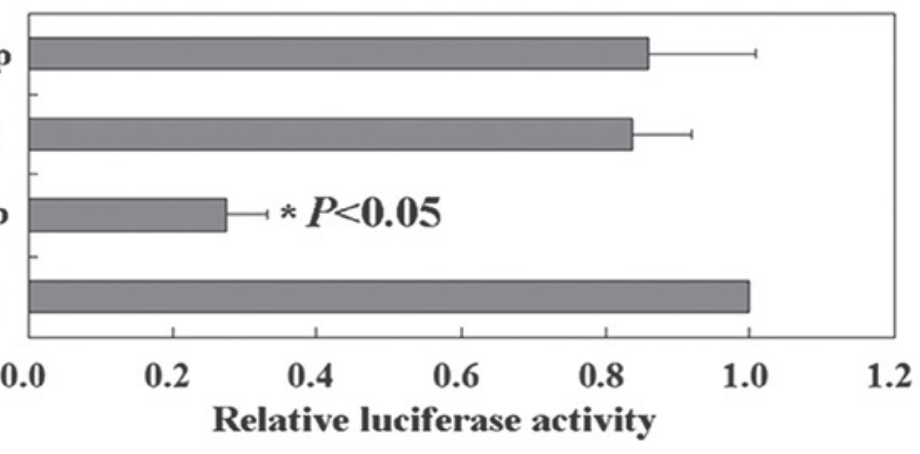

Figure 3. RUNX2 is a target of $m i R-338-3 p$. (A) Predicted $m i R-338-3 p$-binding sequences in the 3 'UTR region of $R U N X 2$. (B) Calculated free energy for hybridization of the RUNX2 3'UTR and $m i R-338-3 p$ (red indicates $R U N X 2$; green indicates $m i R-338-3 p$ ). (C) Association between $R U N X 2$ and $m i R-338-3 p$, detected using a dual-luciferase reporter assay. Data are presented as the mean \pm standard deviation. " $\mathrm{P}<0.05$, vs. the control group. miR, microRNA; RUNX2, runt-related transcription factor 2; UTR, untranslated region; WT, wild-type; Mut, mutant; NC, negative control.

untreated group and the OVX + RESV groups (Tables I and II), while the uterus weight was significantly lower in the OVX group compared with the OVX + RESV group (Table III).
The body weights and levels of subcutaneous fat decreased significantly in the ratstreated with RESV compared with those in the OVX-only group (Tables I and II). 
A

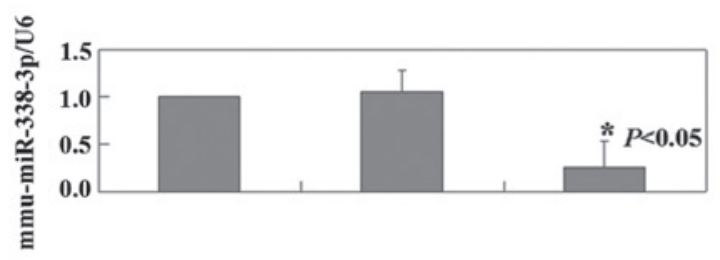

C

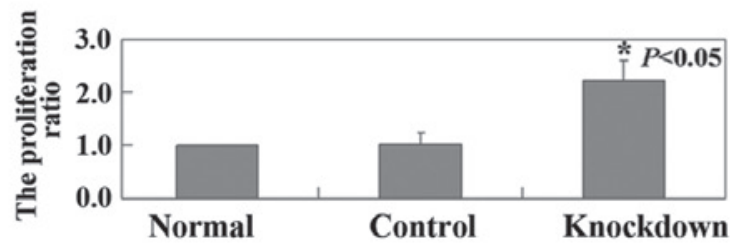

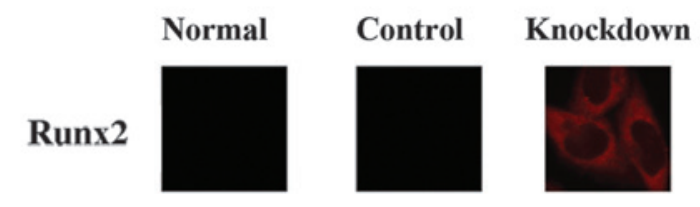

D
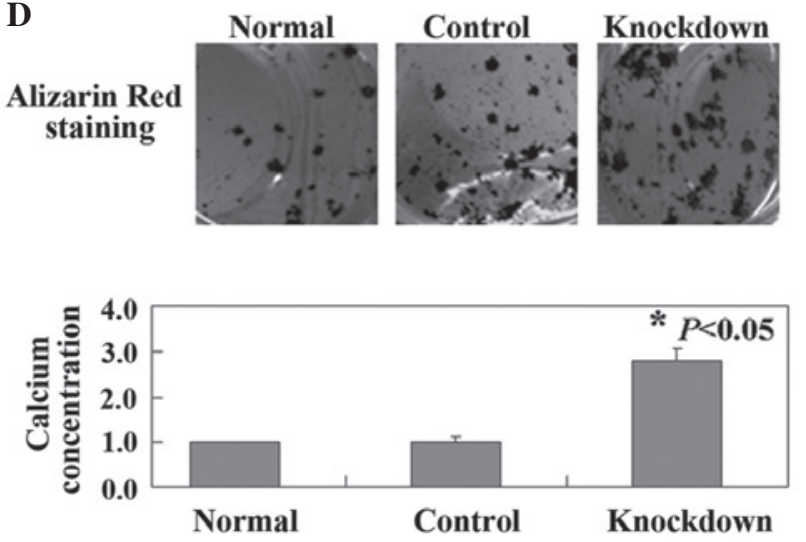

Figure 4. Levels of miR-338-3p and RUNX2 were measured in the HOB cells following mir-338-3p knockdown. (A) mRNA expression levels of $m i R-338-3 p$ were determined by quantitative polymerase chain reaction. U6 was used as an internal control. (B) Detection of RUNX2 in the HOB cells by immunofluorescence. Red represents RUNX2 protein (magnification, x200). (C) Cell proliferation was assessed using a 3-(4, 5-dimethylthiazol-2-yl)-5-(3-carboxymethoxy phenyl)-2-(4-sulfophenyl)-2H-tetrazolium assay. (D) Mineralization was assessed using Alizarin Red staining, to quantify the levels of extracellular calcium (magnification, $\mathrm{x} 40$ ). Data are presented as the mean \pm standard deviation. ${ }^{\mathrm{P}}<0.05$, vs. the control group. miR, microRNA; RUNX2, runt-related transcription factor 2; HOB, human osteoblast.

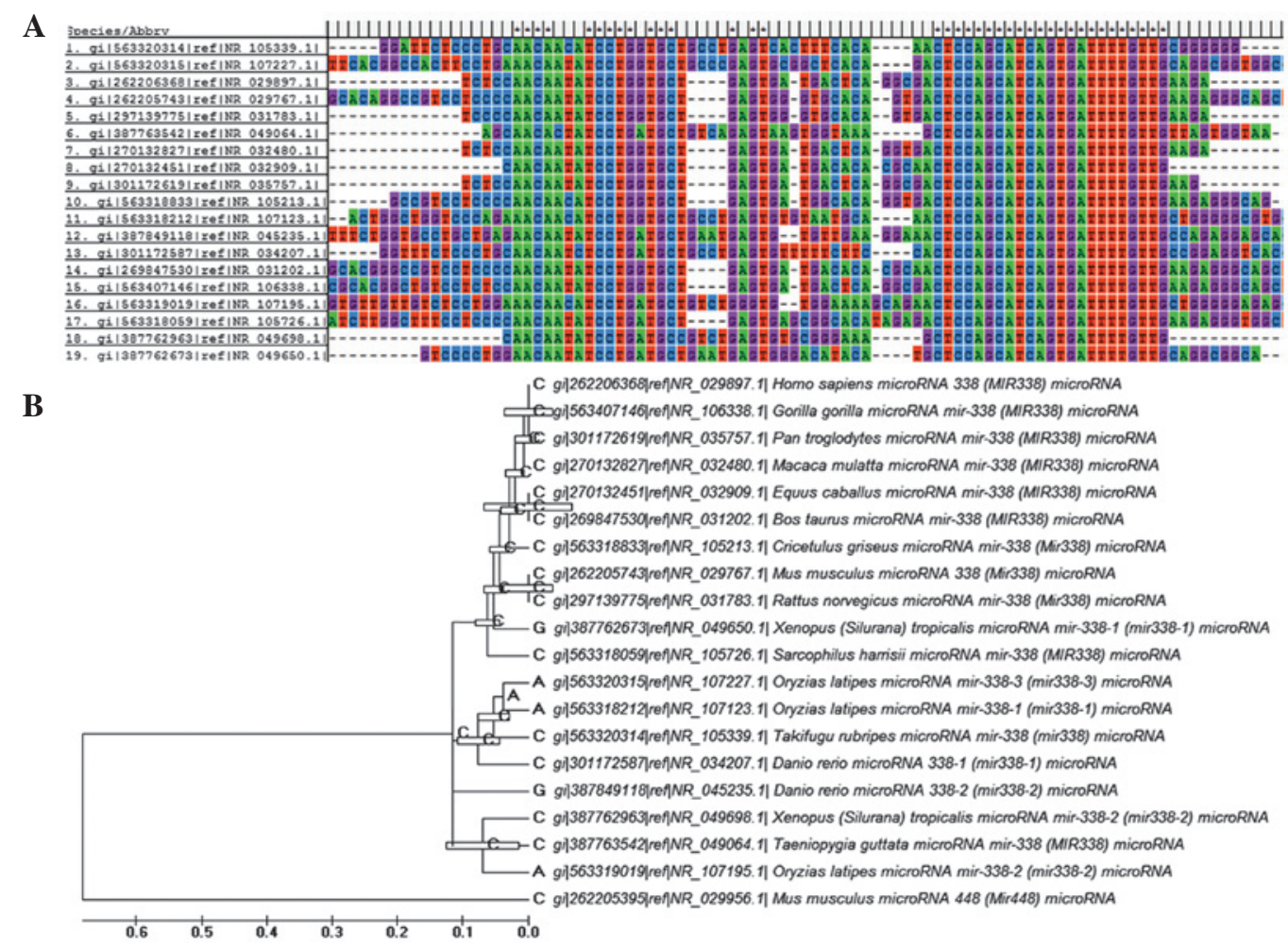

Figure 5. Phylogeny and multiple alignment of $m i R-338-3 p$. (A) Conserved active site motifs of $m i R-338-3 p$ from evolutionary distinct supergroups. (B) An evolutionary tree of $m i R-338-3 p$, generated by maximum likelihood analysis. miR, microRNA.

Bone turnover markers. The BMD was significantly lower in the OVX group compared with the untreated group, and OVX + RESV increased the BMD (Table IV). When comparing the OVX group to the untreated group, the calcium/phosphate ratio and the levels of serum calcium were significantly lower, however, the levels of serum ALP, urinary calcium excretion and urinary DPD/creatinine were significantly higher (Tables V-X). The rats treated with RESV had significantly 
increased levels of serum osteocalcin and calcium/phosphate ratios, however, they exhibited decreased levels of urinary calcium and DPD excretion compared with the rats in the OVX group (Tables V-X). The serum calcium level to increase and the levels of serum ALP and serum phosphorus tended to decrease in the rats that treated with RESV (Tables V-X).

RESV downregulates the expression of miR-338-3p in OVX rats. To identfiy miRNAs, which may have been involved in RESV-induced differentiation in the OVX group, array analysis was used to screen for miRNAs, which were differentially expressed between the OVX group and the OVX + RESV group (Fig. 1). Changes of several miRNAs were identified, of which miR-338-3p was examined in the present study as it exhibited the most marked change in expression. qPCR analysis confirmed that miR-338-3p was significantly decreased in the OVX + RESV treatment compared with the OVX group (Fig. 2A). Furthermore, qPCR and western blot analysis were performed in order to determine the mRNA and protein levels of RUNX2 in each group, respectively (Fig. 2B and C). The mRNA expression of RUNX2 was negatively correlated with that of miR-338-3p (rs=-0.7518; P<0.05; Fig. 2D).

RUNX2 is a direct target of $m i R-338-3 p$. TargetScan was used to identify the potential targets of miR-338-3p. Runx2 was identified as a target of miR-338-3p (Fig. 3A). BibiServ analysis determined the free energy for the RUNX2 3'UTR region and miR-338-3p hybrid was $\sim-11.2 \mathrm{kcal} / \mathrm{mol}$ (Fig. 3B). The relative luciferase activity results revealed that the RUNX2 reporter was suppressed by miR-338-3p (Fig. 3C). These results demonstrated that miR-338-3p specifically targeted the 3'UTR region of RUNX2.

Inhibition of endogenous miR-338-3p induces the proliferation and differentiation of $H O B$ cells. The present study also investigated the effects of miR-338-3p knockdown in HOB cells, by transfection of the HOB cells with miR-338-3p inhibitor. As shown in Fig. 4A, the results of qPCR confirmed the downregulation of endogenous miR-338-3p in the HOB cells following transfection $(\mathrm{P}<0.05)$. Immunofluorescence analysis revealed an increase in the expression of RUNX2 in the HOB cells following transfection (Fig. 4B). Compared with the untransfected cells, a significant increase was observed in the proliferation rate of the HOB cells $(\mathrm{P}<0.05$; Fig. 4C). miR-338-3p knockdown also promoted in vitro calcium deposition in the HOB cells (Fig. 4D). Finally, multiple sequence alignment and phylogenetic analysis were performed, the results of which confirmed miR-338-3p as is a highly conserved microRNA (Fig. 5A and B).

\section{Discussion}

RUNX2 is an essential transcription factor, which controls bone and tooth development by regulating osteoblast and odontoblast differentiation (12-14). RUNX2 is important in mediating the bone morphogenetic protein and transforming growth factor $\beta$ pathways, which are important for osteoblast development and growth (15). In the present study, RUNX2 was found to increase the proliferation and induce the differentiation of HOB cells.
miR-338-3p, an intronic miRNA, is located within the eighth intron (intron-8) of the apoptosis-associated tyrosine kinase gene (16). Liu et al (17) observed that miR-338-3p serves as a negative regulator of osteogenic differentiation in bone marrow stromal cells. Consistent with the previous study, the present study also confirmed that the expression of miR-338-3p was decreased during osteoblast differentiation. Furthermore, the present study found that miR-338-3p knockdown upregulated RUNX2 at the mRNA and protein levels. Bioinformatics analysis indicated that RUNX2 may be a putative target gene of miR-338-3p. Liu et al (17) and Sun et al (18) previously confirmed RUNX2 as a direct target of miR-338-3p.

Following OVX, the rate of bone turnover is known to increase (19). The results of the present study consistently demonstrated that OVX rats exhibited a decrease in serum levels of ALP and OC. Mizutani et al (20) found that RESV attenuates ovariectomy-induced hypertension and bone loss in OVX rats. Casarin et al (21) also found that RESV improves bone repair by modulating levels of the bone morphogenetic protein and osteopontin gene expression in rats.

The present study revealed an intact mechanism by which RESV acts in OVX rats. The results suggested that RESV suppressed miR-338-3p, followed by an increase in the expression of RUNX2 in HOB cells.

\section{References}

1. Guo JD, Li L, Shi YM, Wang HD and Hou SX: Hydrogen water consumption prevents osteopenia in ovariectomized rats. $\mathrm{Br}$ J Pharmacol 168: 1412-1420, 2013.

2. Das S and Crockett JC: Osteoporosis - a current view of pharmacological prevention and treatment. Drug Des Devel Ther 7: 435-448, 2013.

3. Yang Y, Wang B, Fei Q, et al: Validation of an osteoporosis self-assessment tool to identify primary osteoporosis and new osteoporotic vertebral fractures in postmenopausal Chinese women in Beijing. BMC Musculoskelet Disord 14: 271, 2013.

4. Pervaiz S: Resveratrol: from grapevines to mammalian biology. FASEB J 17: 1975-1985, 2003.

5. Baur JA and Sinclair DA: Therapeutic potential of resveratrol: the in vivo evidence. Nat Rev Drug Discov 5: 493-506, 2006.

6. Karuppagounder SS, Pinto JT, Xu H, Chen HL, Beal MF and Gibson GE: Dietary supplementation with resveratrol reduces plaque pathology in a transgenic model of Alzheimer's disease. Neurochem Int 54: 111-118, 2009.

7. Mizutani K, Ikeda K, Kawai Y and Yamori Y: Resveratrol stimulates the proliferation and differentiation of osteoblastic MC3T3-E1 cells. Biochem Biophys Res Commun 253: 859-863, 1998.

8. Liu ZP, Li WX, Yu B, et al: Effects of trans-resveratrol from Polygonum cuspidatum on bone loss using the ovariectomized rat model. J Med Food 8: 14-19, 2005.

9. Calin GA and Croce CM: MicroRNAs and chromosomal abnormalities in cancer cells. Oncogene 25: 6202-6210, 2006.

10. Kiesslich T, Alinger B, Wolkersdörfer GW, Ocker M, Neureiter D and Berr F: Active Wnt signalling is associated with low differentiation and high proliferation in human biliary tract cancer in vitro and in vivo and is sensitive to pharmacological inhibition. Int J Oncol 36: 49-58, 2010.

11. Thompson JD, Higgins DG and Gibson TJ: CLUSTAL W: improving the sensitivity of progressive multiple sequence alignment through sequence weighting, position-specific gap penalties and weight matrix choice. Nucleic Acids Res 22: 4673-4680, 1994.

12. D'Souza RN, Aberg T, Gaikwad J, et al: Cbfa1 is required for epithelial-mesenchymal interactions regulating tooth development in mice. Development 126: 2911-2920, 1999.

13. Miyazaki T, Kanatani N, Rokutanda S, et al: Inhibition of the terminal differentiation of odontoblasts and their transdifferentiation into osteoblasts in Runx 2 transgenic mice. Arch Histol Cytol 71: 131-146, 2008. 
14. Li S, Kong H, Yao N, et al: The role of runt-related transcription factor 2 (Runx2) in the late stage of odontoblast differentiation and dentin formation. Biochem Biophys Res Commun 410 698-704, 2011.

15. Lee KS, Kim HJ, Li QL, et al: Runx2 is a common target of transforming growth factor beta1 and bone morphogenetic protein 2 and cooperation between Runx 2 and Smad5 induces osteoblast-specific gene expression in the pluripotent mesenchymal precursor cell line C2C12. Mol Cell Biol 20: 8783-8792, 2000

16. Rodriguez A, Griffiths-Jones S, Ashurst JL and Bradley A: Identification of mammalian microRNA host genes and transcription units. Genome Res 14: 1902-1910, 2004.

17. Liu H, Sun Q, Wan C,Li L, Zhang L and Chen Z: MicroRNA-338-3p regulates osteogenic differentiation of mouse bone marrow stromal stem cells by targeting Runx2 and Fgfr2. J Cell Physiol 229: 1494-1502, 2014
18. Sun Q, Liu H, Lin H, Yuan G, Zhang L and Chen Z: MicroRNA-338-3p promotes differentiation of mDPC6T into odontoblast-like cells by targeting Runx2. Mol Cell Biochem 377: 143-149, 2013.

19. Govoni KE, Wergedal JE, Chadwick RB, Srivastava AK and Mohan S: Prepubertal OVX increases IGF-1 expression and bone accretion in C57BL/6 mice. Am J Physiol Endocrinol Metab 295: E1172-E1180, 2008

20. Mizutani K, Ikeda K, Kawai Y and Yamori Y: Resveratrol attenuates ovariectomy-induced hypertension and bone loss in stroke-prone spontaneously hypertensive rats. J Nutr Sci Vitaminol (Tokyo) 46: 78-83, 2000.

21. Casarin RC, Casati MZ, Pimentel SP, et al: Resveratrol improves bone repair by modulation of bone morphogenetic proteins and osteopontin gene expression in rats. Int J Oral Maxillofac Surg 43: 900-906, 2014. 